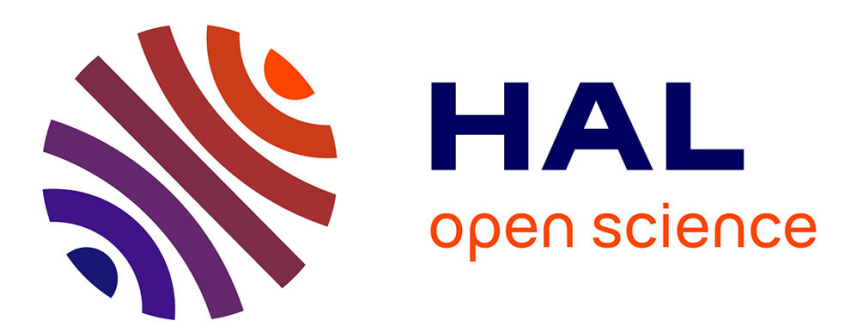

\title{
A modified volume integral equation for anisotropic elastic or conducting inhomogeneities. Unconditional solvability by Neumann series
}

Marc Bonnet

\section{- To cite this version:}

Marc Bonnet. A modified volume integral equation for anisotropic elastic or conducting inhomogeneities. Unconditional solvability by Neumann series. Journal of Integral Equations and Applications, 2017, 29, pp.271-295. hal-01417944

\author{
HAL Id: hal-01417944 \\ https://hal.science/hal-01417944
}

Submitted on 16 Dec 2016

HAL is a multi-disciplinary open access archive for the deposit and dissemination of scientific research documents, whether they are published or not. The documents may come from teaching and research institutions in France or abroad, or from public or private research centers.
L'archive ouverte pluridisciplinaire HAL, est destinée au dépôt et à la diffusion de documents scientifiques de niveau recherche, publiés ou non, émanant des établissements d'enseignement et de recherche français ou étrangers, des laboratoires publics ou privés. 


\title{
A MODIFIED VOLUME INTEGRAL EQUATION FOR ANISOTROPIC ELASTIC OR CONDUCTING INHOMOGENEITIES. UNCONDITIONAL SOLVABILITY BY NEUMANN SERIES
}

\author{
MARC BONNET
}

\begin{abstract}
This work addresses the solvability and solution of volume integrodifferential equations (VIEs) associated with 3D free-space transmission problems (FSTPs) involving elastic or conductive inhomogeneities. A modified version of the singular volume integral equation (SVIE) associated with the VIE is introduced and shown to be of second kind involving a contraction operator, i.e. solvable by Neumann series, implying the wellposedness of the initial VIE. Then, the solvability of VIEs for frequency-domain FSTPs (modelling the scattering of waves by compactly-supported inhomogeneities) follows by a compact perturbation argument. This approach extends work by Potthast (1999) on 2D electromagnetic problems (transverse-electric polarization conditions) involving orthotropic inhomogeneities in a isotropic background, and contains recent results on the solvability of Eshelby's equivalent inclusion problem as special cases. The proposed modified SVIE is also useful for iterative solution methods, as Neumannn series converge (i) unconditionally for static problems and (ii) on some inhomogeneity configurations for which divergence occurs with the usual SVIE for wave scattering problems.
\end{abstract}

1. Introduction. Volume integral equations (VIEs), also known as Lippmann-Schwinger integral equations, arise naturally when considering penetrable inhomogeneities embedded in a homogeneous background medium (for which a fundamental solution is explicitly known). Their derivation and use in e.g. acoustics, elastodynamics or electromagnetism goes back several decades. Since their geometrical support is confined to the spatial region where material properties differ from the background, VIEs are in particular useful for deriving asymptotic or homogenized models. By directly linking remote measurements to unknown inhomogeneities, they also provide a convenient forward modeling approach for medium imaging inverse problems. However, whereas the theory of boundary integral equations is extensively documented, the mathematical properties of VIEs have undergone a comparatively modest coverage, much of it pertaining to electromagnetic scattering problems.

In a previous work [1], to which we refer the reader for bibliography related to the above opening remarks, we established the solvability of a VIE formulation for anisotropic elastodynamic scattering. Therein the VIE problem for an imaginary frequency was shown to be coercive, the real-frequency scattering problems of interest then being found to be compact perturbations of the former.

This work also addresses the solvability and solution of VIEs associated with free-space transmission problems (FSTPs) involving elastic or conductive inhomogeneities, but from a quite different viewpoint. Here, we focus on the singular volume integral equation (SVIE) governing (in the elastic equilibrium case) the unknown initial stress that produces a displacement field identical to that solving the FSTP (Eshelby's equivalent inclusion method, see [3, 12]). We introduce a simple transformation of the SVIE and show the resulting modified SVIE to be of second kind involving a contraction operator, i.e. solvable by Neumann series, which implies the well-posedness of the initial VIE formulation of the static FSTP. Then, the VIEs for frequency-domain FSTPs (modelling the scattering of waves by compactly-supported inhomogeneities) involve integral operators that are compact perturbations of those in corresponding

1991 AMS Mathematics subject classification. 35J15, 45F15, 74J20, 65R20.

Keywords and phrases. Volume integral equation, anisotropy, contraction, Neumann series.

Received by the editors December 16, 2016. 
zero-frequency VIEs, so their solvability follows from uniqueness results on the scattering problem. This approach was initially proposed by Potthast [16] for 2D electromagnetic problems (transverse-electric polarization conditions) involving orthotropic inhomogeneities in a isotropic background. The purpose of this work is to extend Potthast's approach to 3D anisotropic elasticity and potential transmission problems. A similar but distinct transformation of VIEs have been proposed in [15] and companion papers for electromagnetic wave scattering by lossy inhomogeneities, with Neumann series shown to be always convergent; that treatment however is inoperative for lossless materials such as those considered here.

In addition to providing a direct means for establishing the well-posedness of VIE formulations for zero-frequency problems involving inhomogeneities (and the induced well-posedness of VIEs for wave scattering problems), the proposed approach has bearing on iterative solution methods: the underlying restructuration of the VIE formulation makes convergence of Neumann series guaranteed for static problems, and improved for wave scattering problems. Regarding the latter point, we show that there exist inhomogeneity configurations for which Neumann series are convergent for the modified SVIE while diverging for the original SVIE.

This work also extends results in recent investigations on Eshelby's equivalent inclusion method [3], in particular [4, 9] (addressing the existence of solutions for Eshelby's method in the case of ellipsoidal inhomogeneities) and [5] (where the solvability of the singular VIE for isotropic inhomogeneity and background materials is established using Mikhlin's theory of singular integral operators [11]).

This article is organised as follows. The 3D elastostatic FSTP is recast in Section 2 as a second-kind SVIE involving a (singular) contraction operator, leading to the main solvability result (Theorem 1). The corresponding result for 3D potential problems involving anisotropic conductivities (Theorem 2) is (more concisely) established in Sec. 3. The induced well-posedness of VIE formulations for the scattering of waves is then briefly discussed in Sec. 4. The existence of scatterer configurations such that fixed-point iterations are convergent if applied to the modified SVIEs but divergent for the standard version of the SVIE (Prop. 1) is then shown in Sec. 5. Finally, Section 6 collects auxiliary proofs.

2. Elastostatic free-space transmission problem. An inhomogeneity occupying a bounded domain $B \subset \mathbb{R}^{3}$ is embedded in an unbounded homogeneous background medium, with the unbounded complement $\mathbb{R}^{3} \backslash \bar{B}$ of $B$ assumed to be connected. The background and inhomogeneity materials are both assumed to be linearly elastic. Their possibly-anisotropic elastic properties are characterized by their respective fourth-order elasticity tensors $\mathcal{C} \in \mathbb{R}^{3 \times 3 \times 3 \times 3}$ and $\mathcal{C}^{\star} \in L^{\infty}\left(B ; \mathbb{R}^{3 \times 3 \times 3 \times 3}\right)$. They satisfy the usual symmetry properties (i.e. $\mathcal{C}_{i j k \ell}=\mathcal{C}_{i j \ell k}=\mathcal{C}_{k \ell i j}$ and likewise for $\mathcal{C}^{\star}$ ) and the strong ellipticity condition (i.e. $\boldsymbol{\xi}: \mathcal{C}: \boldsymbol{\xi}>0$ and $\boldsymbol{\xi}: \boldsymbol{C}^{\star}(\boldsymbol{x}): \boldsymbol{\xi}>c_{0}|\boldsymbol{\xi}|^{2}>0$ for almost all $\boldsymbol{x} \in B$ and all $\boldsymbol{\xi} \in \mathbb{R}_{\mathrm{sym}}^{3 \times 3}$ ). The boundedness and positivity assumptions exclude the cases of (a) incompressible materials and (b) $B$ being a void. We also let $\Delta \mathcal{C}:=\mathcal{C}^{\star}-\mathcal{C}$ denote the elasticity tensor perturbation in $B$.

The primary field variable is the vector-valued displacement. For a given displacement field $\boldsymbol{w}$, we denote for convenience its restrictions to $B$ and $\mathbb{R}^{3} \backslash \bar{B}$ by $\boldsymbol{w}^{-}$and $\boldsymbol{w}^{+}$, respectively. The stress tensor $\boldsymbol{\sigma}[\boldsymbol{w}]$ is then given by $\boldsymbol{\sigma}[\boldsymbol{w}]=\mathcal{C}^{\star}: \boldsymbol{\varepsilon}\left[\boldsymbol{w}^{-}\right]$in $B$ and by $\boldsymbol{\sigma}[\boldsymbol{w}]=\mathcal{C}: \varepsilon\left[\boldsymbol{w}^{+}\right]$in $\mathbb{R}^{3} \backslash \bar{B}$, where $\boldsymbol{\varepsilon}[\boldsymbol{w}]:=\frac{1}{2}\left(\boldsymbol{\nabla} \boldsymbol{w}+\boldsymbol{\nabla} \boldsymbol{w}^{\mathrm{T}}\right)$ is the linearized strain tensor associated with $\boldsymbol{w}$. The tractions $\boldsymbol{t}[\boldsymbol{w}]$ and $\boldsymbol{t}^{\star}[\boldsymbol{w}]$ exerted by the background and inhomogeneity on the interface $\partial B$, respectively, are then defined (with $\boldsymbol{n}$ denoting the unit normal to $\partial B$ pointing outwards of $B$ and $\left.(\cdot)\right|_{ \pm}$ indicating limiting values) by

$$
\boldsymbol{t}\left[\boldsymbol{w}^{+}\right](\boldsymbol{x})=\left(\mathcal{C}:\left.\varepsilon[\boldsymbol{w}]\right|_{+}\right) \cdot \boldsymbol{n}(\boldsymbol{x}), \quad \boldsymbol{t}^{\star}\left[\boldsymbol{w}^{-}\right](\boldsymbol{x})=\left(\mathcal{C}^{\star}:\left.\varepsilon[\boldsymbol{w}]\right|_{-}\right) \cdot \boldsymbol{n}(\boldsymbol{x}), \quad \boldsymbol{x} \in \partial B
$$


In (1) and hereinafter, symbols '.' and ': ' denote single and double inner products, e.g. $(\boldsymbol{\sigma} \cdot \boldsymbol{n})_{i}=\sigma_{i j} n_{j}$ and $\left(\mathcal{C}^{\star}: \varepsilon\right)_{i j}=\mathcal{C}_{i j k \ell}^{\star} \varepsilon_{k \ell}$, with Einstein's convention of summation over repeated indices implicitly used throughout and component indices always referring to an orthonormal frame.

We consider FSTPs defined as follows: given a background displacement field $\boldsymbol{u} \in H_{\text {loc }}^{1}\left(\mathbb{R}^{3} ; \mathbb{R}^{3}\right)$, find the displacement field $\boldsymbol{u}_{B}$ such that

$$
\begin{array}{rlrl}
\operatorname{div}\left(\mathcal{C}: \varepsilon\left[\boldsymbol{u}_{B}^{+}\right]\right) & =\operatorname{div}(\mathcal{C}: \varepsilon[\boldsymbol{u}]) & & \text { in } \mathbb{R}^{3} \backslash \bar{B}, \\
\operatorname{div}\left(\mathcal{C}^{\star}: \varepsilon\left[\boldsymbol{u}_{B}^{-}\right]\right) & =\operatorname{div}(\mathcal{C}: \varepsilon[\boldsymbol{u}]) & & \text { in } B, \\
\boldsymbol{u}_{B}^{+}(\boldsymbol{\xi})-\boldsymbol{u}(\boldsymbol{\xi}) & =O\left(|\boldsymbol{\xi}|^{-2}\right) & & |\boldsymbol{\xi}| \rightarrow \infty, \\
\boldsymbol{u}_{B}^{+}=\boldsymbol{u}_{B}^{-}, \quad \boldsymbol{t}\left[\boldsymbol{u}_{B}^{+}\right]=\boldsymbol{t}^{\star}\left[\boldsymbol{u}_{B}^{-}\right] & & \text {on } \partial B .
\end{array}
$$

Note that no assumption other than local $H^{1}$ regularity is made on the background field $\boldsymbol{u}$; in particular it is not assumed to verify $\operatorname{div}(\mathcal{C}: \varepsilon[\boldsymbol{u}])=\mathbf{0}$.

2.1. VIE formulation of the elastostatic FSTP. Let $\boldsymbol{G}=\boldsymbol{e}_{k} \otimes \boldsymbol{G}^{k}$ be the elastostatic fundamental tensor for the unbounded background medium, with each vector $\boldsymbol{G}^{k}$ defined as the decaying displacement field solving

$$
-\operatorname{div}\left(\mathcal{C}: \varepsilon\left[\boldsymbol{G}^{k}\right]\right)=\delta \boldsymbol{e}_{k}
$$

( $\delta$ being the Dirac distribution supported at the origin). $\boldsymbol{G}$ is given in inverse Fourier integral form (see [10, Thm. 6.8] and [12]) by

$$
\boldsymbol{G}(\boldsymbol{r})=\frac{1}{4 \pi^{2}} \lim _{R \rightarrow \infty} \int_{B_{R}} \boldsymbol{N}(\boldsymbol{\rho}) e^{\mathrm{i} 2 \pi \boldsymbol{r} \cdot \boldsymbol{\rho}} \mathrm{d} V(\boldsymbol{\rho}), \quad \boldsymbol{r} \in \mathbb{R}^{3} \backslash\{\mathbf{0}\},
$$

where $\boldsymbol{N}(\boldsymbol{\rho})=\boldsymbol{K}(\boldsymbol{\rho})^{-1}(\boldsymbol{K}(\boldsymbol{\rho})$ being the (symmetric, positive definite) acoustic tensor defined by $\left.K_{i k}(\boldsymbol{\rho})=C_{i j k \ell} \rho_{j} \rho_{\ell}[\mathbf{1 2}]\right)$ and $B_{R}$ is the ball of radius $R$ centered at the coordinate origin. With these definitions and conventions, and in view of the translational invariance of the homogeneous full space, $G_{i j}(\boldsymbol{y}-\boldsymbol{x})$ is the $j$-th component of the displacement at $\boldsymbol{y}$ resulting from a unit point force applied at $\boldsymbol{x}$ along the $i$-th direction. The fundamental tensor $\boldsymbol{G}$ and its gradients have the well-known property of being positively homogeneous functions: for any $\boldsymbol{r} \in \mathbb{R}^{3} \backslash\{\mathbf{0}\}$ and $\lambda>0$, we have

$$
\boldsymbol{G}(\lambda \boldsymbol{r})=\lambda^{-1} \boldsymbol{G}(\boldsymbol{r}), \quad \boldsymbol{\nabla} \boldsymbol{G}(\lambda \boldsymbol{r})=\lambda^{-2} \boldsymbol{\nabla} \boldsymbol{G}(\lambda \boldsymbol{r}), \quad \boldsymbol{\nabla}^{2} \boldsymbol{G}(\lambda \boldsymbol{r})=\lambda^{-3} \boldsymbol{\nabla}^{2} \boldsymbol{G}(\lambda \boldsymbol{r}) .
$$

In particular, $\boldsymbol{G}(\boldsymbol{r})$ and $\boldsymbol{\nabla} \boldsymbol{G}(\boldsymbol{r})$, having respectively $O\left(r^{-1}\right)$ and $O\left(r^{-2}\right)$ singularities at the origin, are both locally summable (tensor-valued) functions. For an isotropic material characterized by its shear modulus $\mu$ and Poisson's ratio $\nu$, we have

$$
\mathcal{C}=2 \mu\left(\frac{\nu}{1-2 \nu} \boldsymbol{I} \otimes \boldsymbol{I}+\boldsymbol{I}\right)
$$

(where $\boldsymbol{I}$ and $\mathcal{I}$ are the second-order identity and the fourth-order identity for symmetric tensors, respectively), and $\boldsymbol{G}$ is the well-known Kelvin solution given by:

$$
\boldsymbol{G}(\boldsymbol{r})=\frac{1}{16 \pi \mu(1-\nu) r}[(3-4 \nu) \boldsymbol{I}+\hat{\boldsymbol{r}} \otimes \hat{\boldsymbol{r}}], \quad \text { with } r=|\boldsymbol{r}| \text { and } \hat{\boldsymbol{r}}=\frac{\boldsymbol{r}}{r} .
$$

Volume potential. Define the volume vector potential $\mathcal{W}$ with density $\boldsymbol{h} \in L_{\text {comp }}^{2}\left(\mathbb{R}^{3} ; \mathbb{R}_{\text {sym }}^{3 \times 3}\right)$ by

$$
\mathcal{W}[\boldsymbol{h}](\boldsymbol{x})=\operatorname{div} \int_{\mathbb{R}^{3}} \boldsymbol{G}(\boldsymbol{x}-\boldsymbol{y}) \cdot \boldsymbol{h}(\boldsymbol{y}) \mathrm{d} V(\boldsymbol{y})=\int_{\mathbb{R}^{3}} \boldsymbol{\nabla} \boldsymbol{G}(\boldsymbol{x}-\boldsymbol{y}) \cdot \boldsymbol{h}(\boldsymbol{y}) \mathrm{d} V(\boldsymbol{y}) .
$$


By virtue of known mapping properties of integrodifferential operators treated as pseudodifferential operators $\left[7\right.$, Thm. 6.1.12], $\mathcal{W}$ is well defined as a $L_{\text {comp }}^{2}\left(\mathbb{R}^{3} ; \mathbb{R}_{\text {sym }}^{3 \times 3}\right) \rightarrow H_{\text {loc }}^{1}\left(\mathbb{R}^{3} ; \mathbb{R}^{3}\right)$ operator. Moreover, setting $D:=\operatorname{supp}(\boldsymbol{h})$, the displacement $\boldsymbol{w}=\mathcal{W}[\boldsymbol{h}]$ is a solution in $\mathbb{R}^{3}$ of

$$
\begin{aligned}
& \text { (a) }-\operatorname{div}(\mathcal{C}: \varepsilon[\boldsymbol{w}])=\left\{\begin{array} { r l } 
{ \operatorname { d i v } \boldsymbol { h } } & { \text { in } D , } \\
{ \mathbf { 0 } } & { \text { in } \mathbb { R } ^ { 3 } \backslash \overline { D } , }
\end{array} \quad \text { (b) } \left\{\begin{array}{c}
\boldsymbol{w}^{+}=\boldsymbol{w}^{-}, \\
\boldsymbol{t}\left[\boldsymbol{w}^{+}\right]=\boldsymbol{t}\left[\boldsymbol{w}^{-}\right]+\boldsymbol{h} \cdot \boldsymbol{n}
\end{array} \quad \text { on } \partial D\right.\right. \\
& \text { (c) } \boldsymbol{w}(\boldsymbol{x})=O\left(|\boldsymbol{x}|^{-2}\right), \quad|\boldsymbol{x}| \rightarrow \infty .
\end{aligned}
$$

(see e.g. [1] for a proof of the same result in the more general case of anisotropic elastodynamics). In passing, eqs. $(9 a, b)$ show that $\mathcal{W}[\boldsymbol{h}]$ can be interpreted as the elastostatic field created in an homogeneous unbounded elastic medium by an initial stress field $\boldsymbol{h}$ supported in a compact region $D$.

Volume integrodifferential equation. Using (9), it is easy to check that a displacement field $\boldsymbol{w}$ satisfying the equation $\boldsymbol{w}=\boldsymbol{u}+\mathcal{W}[\Delta \mathcal{C}: \boldsymbol{\varepsilon}(\boldsymbol{w})]$ solves the FSTP (2a-d). This suggests to reformulate the FSTP as the volume integrodifferential equation (VIE)

$$
\boldsymbol{u}_{B}^{-}-\mathcal{L}_{B}\left[\boldsymbol{u}_{B}^{-}\right]=\boldsymbol{u} \quad \text { in } B
$$

with the integrodifferential operator $\mathcal{L}_{B}$ defined by

$$
\mathcal{L}_{B}[\boldsymbol{w}]:=\mathcal{W}[\Delta \mathcal{C}: \varepsilon(\boldsymbol{w})]
$$

supplemented with the representation formula

$$
\boldsymbol{u}_{B}^{+}=\boldsymbol{u}+\mathcal{W}\left[\Delta \mathcal{C}: \varepsilon\left(\boldsymbol{u}_{B}^{-}\right)\right] \quad \text { in } \mathbb{R}^{3} \backslash \bar{B}
$$

Conversely, any solution of the FSTP $2 \mathrm{a}-\mathrm{d})$ satisfies the VIE $10 \mathrm{a}$ and the representation formula (10c), so that problems $(2 \mathrm{a}-\mathrm{d})$ and $110 \mathrm{a}, \mathrm{c})$ are equivalent (a proof of this statement, similar to that given in [1] for the elastodynamic scattering case, is provided for completeness in Sec. 6.1).

2.2. Reformulation of VIE using a contraction operator, solvability. Investigating the solvability of the VIE (10a) is facilitated by reformulating it as a singular volume integral equation (SVIE) for the unknown $\Delta \mathcal{C}: \varepsilon\left[\boldsymbol{u}_{B}^{-}\right]$, as done recently for studying the equivalent inclusion problem in [5]. To this aim, we first note that the background elasticity tensor admits the decomposition

$$
\mathcal{C}=\mathcal{B}: \mathcal{B}
$$

where the fourth-order tensor $\mathcal{B}$ has the same major and minor symmetries as $\mathcal{C}$ and is, like $\mathcal{C}$, positive definite as a $\mathbb{R}_{\text {sym }}^{3 \times 3} \times \mathbb{R}_{\text {sym }}^{3 \times 3} \rightarrow \mathbb{R}$ quadratic form $(\mathcal{B}$ can for example be found by computing the positive definite square root of the $6 \times 6$ matrix associated with $\mathcal{C}$ using e.g. the Voigt-Mandel notation [6]). For the isotropic case (see (6)), we have

$$
\mathcal{B}=\sqrt{2 \mu}\left[\frac{1}{3}\left(\sqrt{\frac{1+\nu}{1-2 \nu}}-1\right) \boldsymbol{I} \otimes \boldsymbol{I}+\boldsymbol{I}\right] .
$$

Then, applying the operator $\mathcal{B}^{-1}: \Delta \mathcal{C}: \varepsilon$ to the integro-differential equation (10a), the FSTP is reformulated as a second-kind SVIE for the new unknown density $\boldsymbol{h}^{\star}:=\mathcal{B}^{-1}: \Delta \mathcal{C}: \varepsilon\left[\boldsymbol{u}_{B}^{-}\right]$:

$$
\mathcal{A}\left[\boldsymbol{h}^{\star}\right]=\mathcal{B}^{-1}: \Delta \mathcal{C}: \varepsilon[\boldsymbol{u}] \quad \text { in } B
$$

The displacement $\boldsymbol{u}_{B}$ solving the FSTP is then given explicitly in terms of $\boldsymbol{h}^{\star}$ by the representation formula

$$
\boldsymbol{u}_{B}=\boldsymbol{u}+\mathcal{W}\left[\boldsymbol{B}: \boldsymbol{h}^{\star}\right] \quad \text { in } B \cup\left(\mathbb{R}^{3} \backslash \bar{B}\right)
$$


The operator $\mathcal{A}: L^{2}\left(B ; \mathbb{R}_{\text {sym }}^{3 \times 3}\right) \rightarrow L^{2}\left(B ; \mathbb{R}_{\text {sym }}^{3 \times 3}\right)$ in $12 \mathrm{a}$ is defined by

$$
\mathcal{A}[\boldsymbol{h}]=\mathcal{B}^{-1}:(\mathcal{I}-\Delta \mathcal{C}: \mathcal{H})[\mathcal{B}: \boldsymbol{h}]
$$

where the singular integral operator $\mathcal{H}$ is given in terms of the potential $\mathcal{W}$ by

$$
\mathcal{H}=\varepsilon[\mathcal{W}] .
$$

The tensor-valued kernel function $\boldsymbol{H}$ associated with $\mathcal{H}$, whose singularity at the origin is not integrable, is the symmetrized version of $\boldsymbol{\nabla} \nabla \boldsymbol{G}$ (see (8)), consistently with the symmetry of $\boldsymbol{h}^{\star}$ (by assumption) and of $\mathcal{H}\left[\boldsymbol{h}^{\star}\right]$ as second-order tensors. It is given using component notation by

$$
H_{i j k \ell}(\boldsymbol{r})=\frac{1}{4}\left[G_{i k, j \ell}(\boldsymbol{r})+G_{i \ell, j k}(\boldsymbol{r})+G_{j k, i \ell}(\boldsymbol{r})+G_{j \ell, i k}(\boldsymbol{r})\right] \quad\left(\boldsymbol{r} \in \mathbb{R}^{3} \backslash\{\mathbf{0}\}\right) .
$$

Then, the following factorization holds for the operator $\mathcal{A}$, and will play a crucial role:

Lemma 1. The singular integral operator $\mathcal{A}$ defined by 13 admits the factorization

$$
\mathcal{A}=\frac{1}{2}\left(\widetilde{\mathcal{C}}^{\star}+\mathcal{I}\right):(\mathcal{I}-\mathcal{Q})
$$

with $\widetilde{\mathcal{C}}^{\star}:=\mathcal{B}^{-1}: \mathcal{C}^{\star}: \mathcal{B}^{-1}$ and $\mathcal{Q}=\mathcal{Q}_{1}: \mathcal{Q}_{2}$, where the multiplication operator $\mathcal{Q}_{1}$ and the integral operator $\mathcal{Q}_{2}$ are defined by

$$
\mathcal{Q}_{1}=\left(\widetilde{\mathcal{C}}^{\star}+\mathcal{I}\right)^{-1}:\left(\widetilde{\mathcal{C}}^{\star}-\mathcal{I}\right), \quad \mathcal{Q}_{2}=\mathcal{I}+2 \mathcal{B}: \mathcal{H}: \mathcal{B}
$$

Proof. We begin by recasting the integral operator $\mathcal{I}-\Delta \mathcal{C}: \mathcal{H}$ in the form

$$
\mathcal{I}-\Delta \mathcal{C}: \mathcal{H}=\left(\mathcal{I}+\frac{1}{2} \Delta \mathcal{C}: \mathcal{C}^{-1}\right)(\mathcal{I}-\mathcal{K})=\frac{1}{2}\left(\mathcal{I}+\mathcal{C}^{\star}: \mathcal{C}^{-1}\right)(\mathcal{I}-\mathcal{K})
$$

with

$$
\mathcal{K}=\left(\mathcal{I}+\mathcal{C}^{\star}: \mathcal{C}^{-1}\right)^{-1}: \Delta \mathcal{C}:\left(\mathcal{C}^{-1}+2 \mathcal{H}\right)
$$

(which is possible since the positive definiteness of $\mathcal{C}$ and $\mathcal{C}^{\star}$ ensure the invertibility of the tensor $\mathcal{I}+\mathcal{C}^{\star}(\boldsymbol{x}): \mathcal{C}^{-1}$ for any $\left.\boldsymbol{x} \in B\right)$. Using $\mathcal{C}^{\star}=\mathcal{B}: \widetilde{\mathcal{C}}^{\star}: \mathcal{B}$ and $\Delta \mathcal{C}=\mathcal{B}:\left(\widetilde{\mathcal{C}}^{\star}-\mathcal{I}\right): \mathcal{B}$, we then have

$$
\left(\mathcal{I}+\mathcal{C}^{\star}: \mathcal{C}^{-1}\right)^{-1}: \Delta \mathcal{C}=\mathcal{B}:\left(\widetilde{\mathcal{C}}^{\star}+\mathcal{I}\right)^{-1}:\left(\widetilde{\mathcal{C}}^{\star}-\mathcal{I}\right): \mathcal{B}
$$

which, substituted into (16), gives

$$
\mathcal{K}=\mathcal{B}:\left(\widetilde{\mathcal{C}}^{\star}+\mathcal{I}\right)^{-1}:\left(\widetilde{\mathcal{C}}^{\star}-\mathcal{I}\right):(\mathcal{I}+2 \mathcal{B}: \mathcal{H}: \mathcal{B}): \mathcal{B}^{-1}=\mathcal{B}: \mathcal{Q}_{1}: \mathcal{Q}_{2}: \mathcal{B}^{-1}
$$

Finally, substituting the above value of $\mathcal{K}$ into 15 and recalling the definition $(13)$ of $\mathcal{A}$ yields, after some manipulations, the claimed factorization of $\mathcal{A}$.

Using Lemma 1 then yields the following modified version of the singular volume integral equation 12a:

$$
(\boldsymbol{I}-\mathcal{Q}) \boldsymbol{h}^{\star}=2\left(\widetilde{\mathcal{C}}^{\star}+\mathcal{I}\right)^{-1}: \mathcal{B}^{-1}: \Delta \mathcal{C}: \varepsilon[\boldsymbol{u}]
$$

wherein the operator $\mathcal{Q}$ is a contraction:

Lemma 2. The operators $\mathcal{Q}_{1}$ and $\mathcal{Q}_{2}$ introduced in Lemma 1 are bounded $L^{2}\left(\mathbb{R}^{3} ; \mathbb{R}_{\text {sym }}^{3 \times 3}\right) \rightarrow$ $L^{2}\left(\mathbb{R}^{3} ; \mathbb{R}_{\text {sym }}^{3 \times 3}\right)$ operators. Moreover:

$$
\text { (i) }\left\|\mathcal{Q}_{1}\right\|<1, \quad \text { (ii) }\left\|\mathcal{Q}_{2}\right\|=1 .
$$

Consequently, the $L^{2}\left(B ; \mathbb{R}_{\text {sym }}^{3 \times 3}\right) \rightarrow L^{2}\left(B ; \mathbb{R}_{\text {sym }}^{3 \times 3}\right)$ operator $\mathcal{Q}=\mathcal{Q}_{1}: \mathcal{Q}_{2}$ is a contraction: $\|\mathcal{Q}\|<1$. 
Proof. See Sec. 2.3

By a standard Neumann series argument, $\mathcal{I}-\mathcal{Q}$ is therefore invertible with bounded inverse. The multiplication operator $\frac{1}{2}\left(\widetilde{\mathcal{C}}^{\star}+\mathcal{I}\right)$ clearly having the same property $\left(\widetilde{\mathcal{C}}^{\star}\right.$ being positive definite), the governing integral operator $\mathcal{A}: L^{2}\left(B ; \mathbb{R}_{\mathrm{sym}}^{3 \times 3}\right) \rightarrow L^{2}\left(B ; \mathbb{R}_{\mathrm{sym}}^{3 \times 3}\right)$ is also boundedly invertible, implying unique solvability of the SVIE $(12 \mathrm{a})$. Finally, $\boldsymbol{u}_{B}$ is given explicitly by $(12 \mathrm{~b})$, and moreover the known boundedness of $\mathcal{W}: L_{\text {comp }}^{2}\left(\mathbb{R}^{3} ; \mathbb{R}_{\text {sym }}^{3 \times 3}\right) \rightarrow H_{\text {loc }}^{1}\left(\mathbb{R}^{3} ; \mathbb{R}^{3}\right)$ shows that the solution mapping $\boldsymbol{u} \mapsto \boldsymbol{u}_{B}^{-}$is continuous as a $H^{1}(B) \rightarrow H^{1}(B)$ operator. This analysis yields our main result:

Theorem 1. Assume that the background and inhomogeneity elasticity tensors $\mathcal{C}$ and $\mathcal{C}^{\star}$ are both strongly elliptic and bounded. Then:

(a) The integral operator $\mathcal{I}-\mathcal{L}_{B}: H^{1}\left(B ; \mathbb{R}^{3}\right) \rightarrow H^{1}\left(B ; \mathbb{R}^{3}\right)$ of the VIE (10a) is invertible with bounded inverse. In particular, (10a) can be solved in two steps:

1. Solve $(\mathcal{I}-\mathcal{Q}) \boldsymbol{h}^{\star}=2\left(\widetilde{\mathcal{C}}^{\star}+\mathcal{I}\right)^{-1}: \mathcal{B}^{-1}: \Delta \mathcal{C}: \varepsilon[\boldsymbol{u}]$, where $\mathcal{Q}: L^{2}\left(B ; \mathbb{R}_{\text {sym }}^{3 \times 3}\right) \rightarrow L^{2}\left(B ; \mathbb{R}_{\text {sym }}^{3 \times 3}\right)$, defined in Lemma 1 , is a contraction;

2. Evaluate $\boldsymbol{u}_{B}^{-}$using the representation formula $12 \mathrm{~b}$

(b) the transmission problem $\sqrt{2 a}-d)$ has a unique solution $\boldsymbol{u}_{B} \in H_{\text {loc }}^{1}\left(\mathbb{R}^{3} ; \mathbb{R}^{3}\right)$;

(c) The unique solution $\boldsymbol{u}_{B}^{-} \in \bar{H}^{1}\left(B ; \mathbb{R}^{3}\right)$ of the VIE $\left.110 \mathrm{a}\right)$ is the restriction of $\boldsymbol{u}_{B}$ to $B$;

(d) The field $\boldsymbol{u}_{B}^{+}$outside $B$ is given by the integral representation formula (10c).

Proof. Item (a) results from the analysis of this section, in particular Lemmas 1 and 2, Items (b), (c) and (d) are then direct consequences of (a) and the equivalence between the transmission problem and the VIE formulation (see Sec. 2.1).

Remark 1. Transforming the original singular integral equation (12a) using the factorization of Lemma 1 was necessary for the unconditional validity of the Neumann series argument. Indeed, the integral operator $\Delta \mathcal{C}: \mathcal{H}$ involved in $12 \mathrm{a})$ is not always a contraction: since $\mathcal{H}$ is independent on $\Delta \mathcal{C}$, the condition $\|\Delta \mathcal{C}: \mathcal{H}\|<1$ is violated by sufficiently large stiffness perturbations $\Delta \mathcal{C}$ whereas $\|\mathcal{Q}\|<1$ for any $\Delta \mathcal{C}$.

Remark 2. If the background medium occupies a bounded domain $\Omega$ and $\boldsymbol{u}_{B}$ satisfies wellposed boundary conditions on $\partial \Omega$, the VIE (10a) still holds, with $\boldsymbol{G}(\boldsymbol{x}-\boldsymbol{y})$ replaced with the Green's tensor $\boldsymbol{G}_{\Omega}(\boldsymbol{y}, \boldsymbol{x})$ satisfying appropriate homogeneous boundary conditions on $\partial \Omega$. Its well-posedness then stems from the fact that $\mathcal{W}_{\Omega}-\mathcal{W}$ (with $\mathcal{W}_{\Omega}$ denoting the volume potential (8) with $\boldsymbol{G}$ replaced by $\left.\boldsymbol{G}_{\Omega}\right)$ defines a compact $L^{2}\left(\Omega ; \mathbb{R}_{\mathrm{sym}}^{3 \times 3}\right) \rightarrow H^{1}\left(\Omega ; \mathbb{R}^{3}\right)$ operator (its kernel function being non-singular for $\boldsymbol{y}=\boldsymbol{x}$ ).

Remark 3. The solvability of the elastostatic SVIE is established in [5] for the less-general case of isotropic inhomogeneity and background materials, by explicitly computing the symbolic determinant of the singular integral operator and invoking Mikhlin's theory of singular integral operators [11]. Moreover, solvability results for the special case of ellipsoidal inhomogeneities are given in [4, 9].

Remark 4. Lemma 2 provides an alternative proof of the previously-mentioned boundedness of the volume potential $\mathcal{W}$ as a $L_{\text {comp }}^{2}\left(\mathbb{R}^{3} ; \mathbb{R}_{\text {sym }}^{3 \times 3}\right) \rightarrow H_{\text {loc }}^{1}\left(\mathbb{R}^{3} ; \mathbb{R}^{3}\right)$ operator. 
2.3. Proof of Lemma 2. The bound on $\left\|\mathcal{Q}_{1}\right\|$ for $\mathcal{Q}_{1}: L^{2}\left(\mathbb{R}^{3} ; \mathbb{R}_{\mathrm{sym}}^{3 \times 3}\right) \rightarrow L^{2}\left(\mathbb{R}^{3} ; \mathbb{R}_{\mathrm{sym}}^{3 \times 3}\right)$ follows at once from $\mathcal{Q}_{1}=\mathcal{I}-2\left(\widetilde{\mathcal{C}}^{\star}+\mathcal{I}\right)^{-1}$ : the positive definiteness of $\widetilde{\mathcal{C}}^{\star}$ implies that $\mathbf{0}<\left(\widetilde{\mathcal{C}}^{\star}(\boldsymbol{x})+\mathcal{I}\right)^{-1}<\mathcal{I}$, i.e. that $\|\mathcal{Q}(\boldsymbol{x})\|<1$ (as a $\mathbb{R}_{\mathrm{sym}}^{3 \times 3} \rightarrow \mathbb{R}_{\mathrm{sym}}^{3 \times 3}$ linear operator) at any $\boldsymbol{x} \in B$; moreover $\boldsymbol{Q}_{1}(\boldsymbol{x})=\mathbf{0}$ for $\boldsymbol{x} \notin \bar{B}$.

Regarding the bound on $\left\|\mathcal{Q}_{2}\right\|$, we begin by noting some useful properties of the singular integral operator $\mathcal{H}$. The kernel $\boldsymbol{\nabla} \boldsymbol{G}$ defines a tempered distribution (since $\boldsymbol{\nabla} \boldsymbol{G}$ is locally summable and belongs to the class of slowly growing functions), making the convolution $\mathcal{W}[\boldsymbol{h}]=\boldsymbol{\varepsilon}[\boldsymbol{G}] \star \boldsymbol{h}$ well defined for any $\boldsymbol{h} \in L_{\text {comp }}^{2}\left(\mathbb{R}^{3} ; \mathbb{R}_{\text {sym }}^{3 \times 3}\right.$ ) (note that here the convolution $\star$ entails an inner product, i.e. $\varepsilon[\boldsymbol{G}] \star \boldsymbol{h}=\int_{\mathbb{R}^{3}} \boldsymbol{\varepsilon}[\boldsymbol{G}](\cdot-\boldsymbol{y}): \boldsymbol{h}(\boldsymbol{y}) \mathrm{d} V(\boldsymbol{y})$.). Therefore, we also have that $\mathcal{H}[\boldsymbol{h}]=\boldsymbol{H} \star \boldsymbol{h}$ in the sense of distributions. Moreover, under the present conditions, the distributional version of the Fourier convolution theorem applies:

$$
\mathcal{F}[\mathcal{H}(\boldsymbol{h})](\boldsymbol{\rho})=\mathcal{F}[\boldsymbol{H} \star \boldsymbol{h}](\boldsymbol{\rho})=\widehat{\boldsymbol{H}}(\boldsymbol{\rho}): \hat{\boldsymbol{h}}(\boldsymbol{\rho}),
$$

with the Fourier transform $\mathcal{F}$ defined such that $\hat{f}(\boldsymbol{\rho})=\mathcal{F}[f](\boldsymbol{\rho})=\int_{\mathbb{R}^{3}} e^{-2 \pi \mathrm{i} x \cdot \boldsymbol{\rho}} f(\boldsymbol{x}) \mathrm{d} V_{x}$ whenever $f \in L^{1}\left(\mathbb{R}^{3}\right)$. Moreover, using (14) and (4), $\widehat{\boldsymbol{H}}(\boldsymbol{\rho})$ is given for $\boldsymbol{\rho} \neq \mathbf{0}$, in component form, by

$$
\widehat{H}_{i j k \ell}(\boldsymbol{\rho})=-\frac{1}{4}\left[N_{j \ell}(\boldsymbol{\rho}) \rho_{i} \rho_{k}+N_{i \ell}(\boldsymbol{\rho}) \rho_{j} \rho_{k}+N_{j k}(\boldsymbol{\rho}) \rho_{i} \rho_{\ell}+N_{i k}(\boldsymbol{\rho}) \rho_{j} \rho_{\ell},\right],
$$

where $\boldsymbol{N}(\boldsymbol{\rho})$ is again the inverse of the acoustic tensor, see (4). For any $\boldsymbol{h} \in L^{2}\left(\mathbb{R}^{3} ; \mathbb{R}_{\mathrm{sym}}^{3 \times 3}\right)$, we therefore have

$$
\mathcal{F}\left[\mathcal{Q}_{2}(\boldsymbol{h})\right](\boldsymbol{\rho})=(\mathcal{I}+2 \mathcal{B}: \widehat{\boldsymbol{H}}(\hat{\boldsymbol{\rho}}): \mathcal{B}): \hat{\boldsymbol{h}}(\boldsymbol{\rho}) .
$$

Recalling decomposition (11) of $\mathcal{C}$ and defining the third-order tensor $\boldsymbol{D}(\boldsymbol{\rho})$ by $D_{a b c}(\boldsymbol{\rho})=$ $\mathcal{B}_{a b c d} \rho_{d}$, the acoustic tensor components are given by $K_{i k}(\boldsymbol{\rho})=D_{a b i}(\boldsymbol{\rho}) D_{a b k}(\boldsymbol{\rho})$. Using (19), we then have

$$
(\mathcal{B}: \widehat{\boldsymbol{H}}: \mathcal{B})_{i j k \ell}=\mathcal{B}_{i j a b} \hat{H}_{a b c d} \mathcal{B}_{c d k \ell}=-D_{i j a}\left[D_{p q a} D_{p q c}\right]^{-1} D_{k \ell c}
$$

(where the dependence of $\widehat{\boldsymbol{H}}$ and $\boldsymbol{D}$ in $\boldsymbol{\rho}$ is omitted for brevity and, by a slight abuse of notation, $\left[E_{a c}\right]^{-1}$ denotes the ac entry of the matrix $\left.\boldsymbol{E}^{-1}\right)$. Since $D_{i j k}=D_{j i k}, \boldsymbol{D}$ can be set in $6 \times 3$ matrix form (representing for given $\boldsymbol{\rho}$ a $\mathbb{R}_{\mathrm{sym}}^{3 \times 3} \rightarrow \mathbb{R}^{3}$ linear operator), and (20) is equivalent to the matrix equality:

$$
[\mathcal{B}: \widehat{\boldsymbol{H}}: \boldsymbol{B}]_{6 \times 6}=-[\boldsymbol{D}]_{6 \times 3}\left[\boldsymbol{D}^{\mathrm{T}} \boldsymbol{D}\right]_{3 \times 3}^{-1}\left[\boldsymbol{D}^{\mathrm{T}}\right]_{3 \times 6} .
$$

Let $[\boldsymbol{D}]_{6 \times 3}=\boldsymbol{Y} \boldsymbol{\Lambda} \boldsymbol{Z}^{\mathrm{T}}$ denote the singular value decomposition of $[\boldsymbol{D}]_{6 \times 3}$, where $\boldsymbol{Y} \in \mathbb{R}^{6 \times 6}$ and $\boldsymbol{Z} \in \mathbb{R}^{3 \times 3}$ are unitary matrices while the matrix $\boldsymbol{\Lambda} \in \mathbb{R}^{6 \times 3}$ holding the singular values has the form $\boldsymbol{\Lambda}=\left[\begin{array}{ll}\boldsymbol{\lambda} & \mathbf{0}\end{array}\right]^{\mathrm{T}}$, with $\boldsymbol{\lambda} \in \mathbb{R}^{3 \times 3}$ a diagonal matrix with real positive entries. The (positive definite) acoustic tensor is then given by $\boldsymbol{K}=\boldsymbol{K}(\boldsymbol{\rho})=\left[\boldsymbol{D}^{\mathrm{T}} \boldsymbol{D}\right]_{3 \times 3}=\boldsymbol{Z} \boldsymbol{\lambda}^{2} \boldsymbol{Z}^{\mathrm{T}}$, implying the positive definiteness (and invertibility) of $\boldsymbol{\lambda}$, and we find

$$
\begin{aligned}
& {[\mathcal{B}: \widehat{\boldsymbol{H}}: \mathcal{B}]_{6 \times 6}=-\left(\boldsymbol{Y} \boldsymbol{\Lambda} \boldsymbol{Z}^{\mathrm{T}}\right)\left(\boldsymbol{Z} \boldsymbol{\lambda}^{-2} \boldsymbol{Z}^{\mathrm{T}}\right)\left(\boldsymbol{Z} \boldsymbol{\Lambda}^{\mathrm{T}} \boldsymbol{Y}^{\mathrm{T}}\right)=-\boldsymbol{Y}\left(\boldsymbol{\Lambda} \boldsymbol{\lambda}^{-2} \boldsymbol{\Lambda}^{\mathrm{T}}\right) \boldsymbol{Y}^{\mathrm{T}}} \\
& =-\boldsymbol{Y}\left(\left[\begin{array}{ll}
\boldsymbol{\lambda} & \mathbf{0}
\end{array}\right]^{\mathrm{T}} \boldsymbol{\lambda}^{-2}\left[\begin{array}{ll}
\boldsymbol{\lambda} & 0
\end{array}\right]\right) \boldsymbol{Y}^{\mathrm{T}}=-\boldsymbol{Y}\left[\begin{array}{ll}
\boldsymbol{I} & \mathbf{0} \\
\mathbf{0} & \mathbf{0}
\end{array}\right] \boldsymbol{Y}^{\mathrm{T}} .
\end{aligned}
$$

Consequently, we have

$$
[\mathcal{I}+2 \mathcal{B}: \widehat{\boldsymbol{H}}: \mathcal{B}]_{6 \times 6}=\boldsymbol{Y}\left[\begin{array}{cc}
-\boldsymbol{I} & \mathbf{0} \\
\mathbf{0} & \boldsymbol{I}
\end{array}\right] \boldsymbol{Y}^{\mathrm{T}}
$$


implying $|(\mathcal{I}+2 \mathcal{B}: \widehat{\boldsymbol{H}}(\boldsymbol{\rho}): \mathcal{B}) \cdot \hat{\boldsymbol{h}}(\boldsymbol{\rho})|=|\hat{\boldsymbol{h}}(\boldsymbol{\rho})|$ for any $\boldsymbol{\rho}$. With the help of Plancherel's theorem, this in turn yields

$$
\left\|\mathcal{Q}_{2}[\boldsymbol{h}]\right\|_{L^{2}\left(\mathbb{R}^{3} ; \mathbb{R}_{\mathrm{sym}}^{3 \times 3}\right)}=\left\|\widehat{\mathcal{Q}_{2}[\boldsymbol{h}]}\right\|_{L^{2}\left(\mathbb{R}^{3} ; \mathbb{R}_{\mathrm{sym}}^{3 \times 3}\right)}=\|\hat{\boldsymbol{h}}\|_{L^{2}\left(\mathbb{R}^{3} ; \mathbb{R}_{\mathrm{sym}}^{3 \times 3}\right)}=\|\boldsymbol{h}\|_{L^{2}\left(\mathbb{R}^{3} ; \mathbb{R}_{\mathrm{sym}}^{3 \times 3}\right)}
$$

i.e. that $\left\|\mathcal{Q}_{2}\right\|=1$ as a $L^{2}\left(\mathbb{R}^{3} ; \mathbb{R}_{\text {sym }}^{3 \times 3}\right) \rightarrow L^{2}\left(\mathbb{R}^{3} ; \mathbb{R}_{\text {sym }}^{3 \times 3}\right)$ linear operator. This implies the bound (ii), and the proof of the Lemma is complete.

3. Conductivity transmission problem. The arguments developed in the previous section for elastostatic FSTPs can also be applied to the simpler case of transmission problems involving (e.g. electrostatic) scalar potentials in media characterized by second-order conductivity tensors. This section thus aims at setting the relevant context and notation and concisely establishing the counterpart of Theorem 1, which is a useful result in its own right.

Retaining the geometrical assumptions made in Sec. 2 for the inhomogeneity support $B$, the inhomogeneity is now characterized by its conductivity tensor $\boldsymbol{C}^{\star} \in L^{\infty}\left(B ; \mathbb{R}_{\text {sym }}^{3 \times 3}\right.$ ) (such that $\boldsymbol{\xi} \cdot \boldsymbol{C}^{\star}(\boldsymbol{x}) \cdot \boldsymbol{\xi}>c_{0}|\boldsymbol{\xi}|^{2}>0$ for almost all $\boldsymbol{x} \in B$ and all $\boldsymbol{\xi} \in \mathbb{R}^{3}$ ), and the background medium by a constant, positive definite conductivity tensor $\boldsymbol{C} \in \mathbb{R}_{\mathrm{sym}}^{3 \times 3}$. The case of isotropic properties corresponds, of course, to scalar conductivities $c, c^{\star}$ such that $\boldsymbol{C}=c \boldsymbol{I}$ and $\boldsymbol{C}^{\star}(\boldsymbol{x})=c^{\star}(\boldsymbol{x}) \boldsymbol{I}$.

We consider FSTPs defined as follows: given a background potential $u \in H_{\text {loc }}^{1}\left(\mathbb{R}^{3}\right)$, find the potential $u_{B}$ such that

$$
\begin{array}{rlrl}
\operatorname{div}\left(\boldsymbol{C} \cdot \boldsymbol{\nabla} u_{B}^{+}\right) & =\operatorname{div}(\boldsymbol{C} \cdot \boldsymbol{\nabla} u) & & \text { in } \mathbb{R}^{3} \backslash \bar{B}, \\
\operatorname{div}\left(\boldsymbol{C}^{\star} \cdot \boldsymbol{\nabla} u_{B}^{-}\right) & =\operatorname{div}(\boldsymbol{C} \cdot \boldsymbol{\nabla} u) & & \text { in } B, \\
u_{B}^{+}(\boldsymbol{\xi})-u(\boldsymbol{\xi}) & =O\left(|\boldsymbol{\xi}|^{-2}\right) & & |\boldsymbol{\xi}| \rightarrow \infty, \\
u_{B}^{+}=u_{B}^{-}, \quad \boldsymbol{n} \cdot \boldsymbol{C} \cdot \boldsymbol{\nabla} u_{B}^{+}=\boldsymbol{n} \cdot \boldsymbol{C}^{\star} \cdot \boldsymbol{\nabla} u_{B}^{-} & & \text {on } \partial B .
\end{array}
$$

Let $G$ be the fundamental solution for the unbounded background medium, which solves

$$
-\operatorname{div}(\boldsymbol{C} \cdot \boldsymbol{\nabla} G)=\delta
$$

Using the decomposition $\boldsymbol{C}=\boldsymbol{B} \cdot \boldsymbol{B}$, where $\boldsymbol{B}$ is the positive definite square root of $\boldsymbol{C}, G$ is given by

$$
G(\boldsymbol{x})=\frac{1}{4 \pi \operatorname{det}(\boldsymbol{B})} \frac{1}{\left|\boldsymbol{B}^{-1} \cdot \boldsymbol{x}\right|} .
$$

If $\boldsymbol{C}=c \boldsymbol{I}$ (isotropic case), we recover of course the well-known expression $G(\boldsymbol{x})=1 /(4 \pi c|\boldsymbol{x}|)$.

The volume potential $\mathcal{W}$, acting on densities $\boldsymbol{h} \in L_{\text {comp }}^{2}\left(\mathbb{R}^{3} ; \mathbb{R}^{3}\right)$, is defined by

$$
\mathcal{W}[\boldsymbol{h}](\boldsymbol{x})=\int_{\mathbb{R}^{3}} \boldsymbol{\nabla} G(\boldsymbol{x}-\boldsymbol{y}) \cdot \boldsymbol{h}(\boldsymbol{y}) \mathrm{d} V(\boldsymbol{y}),
$$

and is a bounded $L_{\text {comp }}^{2}\left(\mathbb{R}^{3} ; \mathbb{R}^{3}\right) \rightarrow H_{\text {loc }}^{1}(\mathbb{R})$ operator. Setting again $D:=\operatorname{supp}(\boldsymbol{h})$, the potential $w=\mathcal{W}[\boldsymbol{h}]$ solves

$$
\begin{gathered}
\text { (a) }-\operatorname{div}(\boldsymbol{C} \cdot \boldsymbol{\nabla} w)=\left\{\begin{aligned}
\operatorname{div} \boldsymbol{h} & \text { in } D, \\
\mathbf{0} & \text { in } \mathbb{R}^{3} \backslash \bar{D},
\end{aligned}\right. \\
\text { (b) }\left.w\right|_{+}=\left.w\right|_{-} \text {and }\left.\boldsymbol{n} \cdot \boldsymbol{C} \cdot \boldsymbol{\nabla} w\right|_{+}=\left.\boldsymbol{n} \cdot \boldsymbol{C}^{\star} \cdot \boldsymbol{\nabla} w\right|_{-}+\boldsymbol{h} \cdot \boldsymbol{n} \quad \text { on } \partial D, \\
\text { (c) } w(\boldsymbol{x})=O\left(|\boldsymbol{x}|^{-2}\right), \quad|\boldsymbol{x}| \rightarrow \infty
\end{gathered}
$$


The FSTP then admits the VIE formulation

$$
\begin{aligned}
u_{B}^{-}-L_{B}\left[u_{B}^{-}\right] & =u, & & \text { in } B, \\
u_{B}^{+} & =u+\mathcal{W}\left[\Delta \boldsymbol{C} \cdot \boldsymbol{\nabla} u_{B}^{-}\right], & & \text {in } \mathbb{R}^{3} \backslash \bar{B},
\end{aligned}
$$

where the integrodifferential operator $L_{B}$ is defined by $L_{B}[w]:=\mathcal{W}[\Delta \boldsymbol{C} \cdot \boldsymbol{\nabla} w]$. Conversely, transposing arguments given earlier for the elastic case, any solution of the FSTP (22a) satisfies the VIE 27a).

Theorem 2. Assume that the background and inhomogeneity conductivity tensors $\boldsymbol{C}$ and $\boldsymbol{C}^{\star}$ are both positive definite and bounded. Then:

(a) The integrodifferential operator $I-L_{B}: H^{1}\left(B ; \mathbb{R}^{3}\right) \rightarrow H^{1}\left(B ; \mathbb{R}^{3}\right)$ is invertible with bounded inverse;

(b) the transmission problem (22a) has a unique solution $u_{B} \in H_{\text {loc }}^{1}\left(\mathbb{R}^{3}\right)$;

(c) The unique solution $u_{B}^{-} \in H^{1}(B)$ of the integrodifferential equation (27a) is the restriction of $u_{B}$ to $B$

(d) The field $u_{B}^{+}$outside $B$ is given by the integral representation formula (27b).

Proof. As for Theorem 1, we only need to prove item (a). Applying the operator $\boldsymbol{B}^{-1} \cdot \Delta \boldsymbol{C} \cdot \boldsymbol{\nabla}$ to the integro-differential equation (27a), we obtain the equivalent problem
(a) $\boldsymbol{A}\left[\boldsymbol{h}^{\star}\right]=\boldsymbol{B}^{-1} \cdot \Delta \boldsymbol{C} \cdot \boldsymbol{\nabla} u$ in $B$,
(b) $u_{B}=u+\mathcal{W}\left[\boldsymbol{B} \cdot \boldsymbol{h}^{\star}\right] \quad$ in $B \cup\left(\mathbb{R}^{3} \backslash \bar{B}\right)$

for the new unknown $\boldsymbol{h}^{\star}:=\boldsymbol{B}^{-1} \cdot \Delta \boldsymbol{C} \cdot \boldsymbol{\nabla} u_{B}^{-}$, and with the singular integral operator $\boldsymbol{A}$ : $L^{2}\left(B ; \mathbb{R}^{3}\right) \rightarrow L^{2}\left(B ; \mathbb{R}^{3}\right)$ defined by

$$
\boldsymbol{A}[\boldsymbol{h}]=\boldsymbol{B}^{-1} \cdot(\boldsymbol{I}-\Delta \boldsymbol{C} \cdot \boldsymbol{\nabla} \mathcal{W})[\boldsymbol{B} \cdot \boldsymbol{h}] .
$$

One readily checks, by inspection, that the factorization

$$
\boldsymbol{A}=\frac{1}{2}\left(\tilde{\boldsymbol{C}}^{\star}+\boldsymbol{I}\right) \cdot\left(\boldsymbol{I}-\boldsymbol{Q}_{1} \cdot \boldsymbol{Q}_{2}\right)
$$

holds, with $\tilde{\boldsymbol{C}}^{\star}:=\boldsymbol{B}^{-1} \cdot \boldsymbol{C}^{\star} \cdot \boldsymbol{B}^{-1}$ and where the multiplication operator $\boldsymbol{Q}_{1}$ and the integral operator $\boldsymbol{Q}_{2}$ are defined by

$$
\boldsymbol{Q}_{1}=\left(\tilde{\boldsymbol{C}}^{\star}+\boldsymbol{I}\right)^{-1} \cdot\left(\tilde{\boldsymbol{C}}^{\star}-\boldsymbol{I}\right), \quad \boldsymbol{Q}_{2}=\boldsymbol{I}+2 \boldsymbol{B} \cdot \boldsymbol{\nabla} \mathcal{W} \cdot \boldsymbol{B} .
$$

Moreover, $\boldsymbol{Q}_{1}$ and $\boldsymbol{Q}_{2}$ are bounded $L^{2}\left(B ; \mathbb{R}^{3}\right) \rightarrow L^{2}\left(B ; \mathbb{R}^{3}\right)$ operators and verify

$$
\text { (i) }\left\|\boldsymbol{Q}_{1}\right\|<1, \quad \text { (ii) }\left\|\boldsymbol{Q}_{2}\right\|=1 \text {. }
$$

The bound (i) holds for the same reasons as in the elastic case (with fourth-order tensors changed to $3 \times 3$ matrices), and a proof for (ii) is given thereafter. Consequently, the $L^{2}\left(B ; \mathbb{R}^{3}\right) \rightarrow L^{2}\left(B ; \mathbb{R}^{3}\right)$ operator $\boldsymbol{Q}_{1} \cdot \boldsymbol{Q}_{2}$ is a contraction: $\left\|\boldsymbol{Q}_{1} \cdot \boldsymbol{Q}_{2}\right\|<1$. The same arguments as for the elastic case then allow to complete the proof of Theorem 2 .

Proof of $\left\|\boldsymbol{Q}_{2}\right\|=1$. As before, the distributional version of the Fourier convolution theorem applies:

$$
\mathcal{F}[\nabla \mathcal{W}(\boldsymbol{h})](\boldsymbol{\rho})=\mathcal{F}[\boldsymbol{H} \star \boldsymbol{h}](\boldsymbol{\rho})=\widehat{\boldsymbol{H}}(\boldsymbol{\rho}) \cdot \hat{\boldsymbol{h}}(\boldsymbol{\rho}),
$$

(having set $\boldsymbol{H}:=\nabla \nabla G$ ) while $\widehat{\boldsymbol{H}}(\boldsymbol{\rho})$ is given (applying the Fourier transform to (23)) by

$$
\widehat{\boldsymbol{H}}(\boldsymbol{\rho})=-(\boldsymbol{\rho} \cdot \boldsymbol{C} \cdot \boldsymbol{\rho})^{-1} \boldsymbol{\rho} \otimes \boldsymbol{\rho} .
$$


In particular, $\boldsymbol{\rho} \mapsto \widehat{\boldsymbol{H}}(\boldsymbol{\rho})$ is $C^{\infty}\left(\mathbb{R}^{3} \backslash\{\mathbf{0}\}\right)$ and homogeneous with degree 0 in $\boldsymbol{\rho}$. For any $\boldsymbol{h} \in L^{2}\left(\mathbb{R}^{3} ; \mathbb{R}^{3}\right)$, we have

$$
\mathcal{F}\left[\boldsymbol{Q}_{2}(\boldsymbol{h})\right](\boldsymbol{\rho})=(\boldsymbol{I}+2 \boldsymbol{B} \cdot \widehat{\boldsymbol{H}}(\boldsymbol{\rho}) \cdot \boldsymbol{B}) \cdot \hat{\boldsymbol{h}}(\boldsymbol{\rho})=\left(\boldsymbol{I}-2 \frac{\boldsymbol{B} \cdot \boldsymbol{\rho}}{|\boldsymbol{B} \cdot \boldsymbol{\rho}|} \otimes \frac{\boldsymbol{B} \cdot \boldsymbol{\rho}}{|\boldsymbol{B} \cdot \boldsymbol{\rho}|}\right) \cdot \hat{\boldsymbol{h}}(\boldsymbol{\rho}),
$$

implying $|(\mathcal{I}-2 \boldsymbol{B} \cdot \widehat{\boldsymbol{H}}(\boldsymbol{\rho}) \cdot \boldsymbol{B}) \cdot \hat{\boldsymbol{h}}(\boldsymbol{\rho})|=|\hat{\boldsymbol{h}}(\boldsymbol{\rho})|$ for any $\boldsymbol{\rho} \in \mathbb{R}^{3} \backslash\{\boldsymbol{0}\}$. Plancherel's theorem finally yields the desired bound $\left\|\boldsymbol{Q}_{2}\right\|=1$ through

$$
\left\|\boldsymbol{Q}_{2}[\boldsymbol{h}]\right\|_{L^{2}\left(\mathbb{R}^{3} ; \mathbb{R}^{3}\right)}=\|\boldsymbol{h}\|_{L^{2}\left(\mathbb{R}^{3} ; \mathbb{R}^{3}\right)} .
$$

4. Solvability of VIE formulations for elastic or acoustic wave scattering problems. Consider the elastodynamic problem where an incident monochromatic wave $\boldsymbol{u}$ (with given angular frequency $\omega$, and with the time-harmonic factor $e^{-i \omega t}$ implicitly understood as usual) is scattered by an inhomogeneity characterized by a perturbation $\Delta \rho=\rho^{\star}-\rho$ of the background mass density $\rho$ in addition to the perturbation $\Delta \mathcal{C}$ of elastic moduli. The elastodynamic fundamental tensor $\boldsymbol{G}_{\omega}=\boldsymbol{e}_{k} \otimes \boldsymbol{G}_{\omega}^{k}$ for the background medium is defined in terms of the radiating solutions of

$$
-\operatorname{div}\left(\mathcal{C}: \varepsilon\left[\boldsymbol{G}^{k}\right]\right)-\rho \omega^{2} \boldsymbol{G}^{k}=\delta \boldsymbol{e}_{k}
$$

(referring the reader to [8] and [13] for details on $\boldsymbol{G}_{\omega}$ and the meaning of "radiating" in the cases of isotropic and anisotropic background, respectively). The (now complex-valued) displacement field $\boldsymbol{u}_{B}$ verifies the VIE

$$
\boldsymbol{u}_{B}^{-}-\mathcal{L}_{B}^{\omega}\left[\boldsymbol{u}_{B}^{-}\right]=\boldsymbol{u}, \quad \text { in } B
$$

for the restriction of $\boldsymbol{u}_{B}$ to $B$, with the integrodifferential operator $\mathcal{L}_{B}^{\omega}$ defined by

$$
\mathcal{L}_{B}^{\omega}[\boldsymbol{w}]:=\mathcal{W}_{\omega}[\Delta \mathcal{C}: \varepsilon(\boldsymbol{w})]+\omega^{2} \mathcal{V}_{\omega}[\Delta \rho \boldsymbol{w}]
$$

In $32 \mathrm{~b}, \boldsymbol{\mathcal { W }}_{\omega}$ is the volume potential defined by (8) with $\boldsymbol{G}$ replaced by $\boldsymbol{G}_{\boldsymbol{\omega}}$, while the additional volume potential $\mathcal{V}$ is given for any $\boldsymbol{g} \in H_{\mathrm{loc}}^{1}\left(\mathbb{R}^{3} ; \mathbb{R}^{3}\right)$ by

$$
\mathcal{V}[\boldsymbol{g}](\boldsymbol{x})=\int_{\mathbb{R}^{3}} \boldsymbol{G}_{\omega}(\boldsymbol{x}-\boldsymbol{y}) \cdot \boldsymbol{g}(\boldsymbol{y}) \mathrm{d} V(\boldsymbol{y}),
$$

Since $\boldsymbol{x} \mapsto \boldsymbol{G}_{\omega}(\boldsymbol{x})$ and $\boldsymbol{x} \mapsto \boldsymbol{\nabla}\left(\boldsymbol{G}_{\omega}-\boldsymbol{G}\right)(\boldsymbol{x})$ are known to be weakly singular and bounded, respectively, at $\boldsymbol{x}=\mathbf{0}, \mathcal{V}$ and $\mathcal{W}_{\omega}-\mathcal{W}$ define compact $H^{1}\left(B ; \mathbb{R}^{3}\right) \rightarrow H^{1}\left(B ; \mathbb{R}^{3}\right)$ operators. The operator $\mathcal{I}-\mathcal{L}_{B}^{\omega}$ is therefore Fredholm with index 0 , and its bounded invertibility then follows from known uniqueness results for scattering by inhomogeneities and the Fredholm alternative. We thus recover the solvability result established for elastodynamic VIEs in [1] using other arguments.

Essentially the same arguments apply to the VIE formulation of scattering problems governed by the Helmholtz equation (or anisotropic versions thereof, investigated e.g. in [2]), as previously done in [16] for 2D electromagnetic scattering by anisotropic inhomogeneities.

5. Fixed-point iterations. In addition to being the crucial ingredient for establishing the well-posedness of the VIE formulation of elastostatic FSTPs, the modified SVIE (18), having the form $(\mathcal{I}-\mathcal{Q}) \boldsymbol{h}^{\star}=\boldsymbol{f}$ with $\|\mathcal{Q}\|<1$, can be solved by fixed-point iterations, i.e. by arbitrarily choosing an initial value $\boldsymbol{h}_{0}^{\star}$ and setting

$$
\boldsymbol{h}_{n+1}^{\star}=\mathcal{Q}: \boldsymbol{h}_{n}^{\star}+\boldsymbol{f} \quad(n=1,2, \ldots) .
$$


The above iterations amount to computing the sequence of partial sums of the Neumann series $(\mathcal{I}-\mathcal{Q})^{-1} \boldsymbol{f}=\boldsymbol{f}+\mathcal{Q}: \boldsymbol{f}+\mathcal{Q}: \mathcal{Q}: \boldsymbol{f}+\ldots$, whose convergence is assured for any inhomogeneity configuration $(B, \Delta \mathcal{C})$, so that $\boldsymbol{h}_{n}^{\star} \rightarrow \boldsymbol{h}^{\star}$ in $L^{2}\left(B ; \mathbb{R}^{3 \times 3}\right)$.

Fixed-point iterations of the form (34) are also commonly applied to the solution of wave scattering problems; they are often referred to in this context as Born series or iterated Born approximations. Fixed-point iterations may be applied to the frequency-domain counterpart of the SVIE in either classical form (12a) or modified form (18), obtained by using the relevant frequency-domain fundamental solution therein. Their convergence is subject to restrictions on the scatterer characteristics and the frequency (the scatterer needing to be "sufficiently weak"). In what follows, we show the existence of scatterer configurations such that fixed-point iterations are convergent if applied to the modified SVIE but divergent if applied to the standard SVIE.

5.1. Scattering of acoustic waves. Consider the scattering of an incident monochromatic acoustic wave $u$ by a penetrable inhomogeneity, with the background and inhomogeneity materials having the same bulk modulus $\mu$ but different mass densities $\rho, \rho^{\star}$ (the background wave velocity being $c=\sqrt{\mu / \rho}$ ). The complex-valued total acoustic pressure $u_{B}$ then satisfies the VIE

$$
u_{B}^{-}-L_{B}^{\omega}\left[u_{B}^{-}\right]=u \quad \text { in } B,
$$

with the integrodifferential operator $L_{B}^{\omega}$ defined (with $r:=-1+\rho / \rho^{\star}=-\Delta \rho / \rho^{\star}$ ) by

$$
L_{B}^{\omega}[w]:=\mathcal{W}_{\omega}[r \nabla w] .
$$

In $35 \mathrm{~b}), \mathcal{W}_{\omega}$ is the volume potential defined by (25) with $G$ replaced by the fundamental solution $G_{\omega}$ of the Helmholtz equation, i.e.:

$$
G_{\omega}(\boldsymbol{r})=\frac{e^{\mathrm{i} k|\boldsymbol{r}|}}{4 \pi|\boldsymbol{r}|}, \quad \text { with } k=\omega \sqrt{\rho / \mu}
$$

Note that the conductivity-like transmission problem in the zero-frequency limiting case is $22 \mathrm{a}$ c) with $\boldsymbol{C}=\boldsymbol{I}$ and $\boldsymbol{C}^{\star}=\left(\rho / \rho^{\star}\right) \boldsymbol{I}$. The case of dissimilar values of $\mu$ would result in an additional, weakly singular, volume integral operator in equation $(35 \mathrm{a})$, similar to $\mathcal{V}_{\omega}$ in $(32 \mathrm{~b})$.

The scattering problem can then be recast in terms of the SVIE

$$
\boldsymbol{h}^{\star}-r \boldsymbol{\nabla} \mathcal{W}_{\omega}\left[\boldsymbol{h}^{\star}\right]=r \boldsymbol{\nabla} u \quad \text { in } B
$$

for the new unknown $\boldsymbol{h}^{\star}:=r \boldsymbol{\nabla} u_{B}^{-}$, obtained by applying the operator $r \boldsymbol{\nabla}$ to (35a). In turn, the operator $r \boldsymbol{\nabla} \mathcal{W}_{\omega}$ can be recast in a factorized form similar to (30), which transforms the singular integral equation 37 into

$$
\boldsymbol{h}^{\star}-\boldsymbol{Q}^{\omega}\left[\boldsymbol{h}^{\star}\right]=\frac{2 r}{r+2} \nabla u \quad \text { in } B, \quad \text { with } \quad \boldsymbol{Q}^{\omega}:=\frac{r}{r+2} \boldsymbol{Q}_{2}^{\omega}, \quad \boldsymbol{Q}_{2}^{\omega}:=\boldsymbol{I}+2 \boldsymbol{\nabla} \mathcal{W}_{\omega} .
$$

The following two lemmas, whose proofs are given in the Appendix, hold true for $\nabla \mathcal{W}_{\omega}$ as a (bounded) $L^{2}\left(B ; \mathbb{R}^{3}\right) \rightarrow L^{2}\left(B ; \mathbb{R}^{3}\right)$ operator:

Lemma 3. There exists a constant $C_{1}>0$, independent of $\omega$ and $B$, such that

$$
\left\|\nabla \mathcal{W}_{\omega}\right\| \geq C_{1}
$$

Lemma 4. Assume that $B \subset B_{R}$, where $B_{R}$ is the ball of radius $R$. We have

$$
\left\|\nabla\left(\mathcal{W}_{\omega}-\mathcal{W}\right)\right\| \leq C_{2}(k R)^{2}
$$


with $C_{2}=2 \pi E$, where $E$ is the smallest constant satisfying inequality (44). The constant $C_{2}$ is independent on $\omega$ and $B$, and is approximately given by $C_{2} \approx 0.61577$.

We then obtain the following result on the conditions for the convergence of fixed-point iterations applied to the singular integral equation in either its classical form (37) or its modified form 38 :

Proposition 1. Set $h(r):=1 / r$ if $r>0$ and $h(r):=-1-1 / r$ if $-1<r<0$. Then:

(a) Fixed-point iterations applied to the integral equation (37) diverge if $r C_{1}>1$;

(b) Fixed-point iterations applied to the integral equation (38) converge if $C_{2}(k R)^{2}<h(r)$.

Proof. Case (a) follows directly from Lemma 3 and the convergence requirement $\left\|r \boldsymbol{\nabla} \mathcal{W}_{\omega}\right\|<1$.

Regarding case (b), we write $\boldsymbol{Q}_{2}^{\omega}=\boldsymbol{Q}_{2}+2 \boldsymbol{\nabla}\left(\mathcal{W}_{\omega}-\mathcal{W}\right)$. Since $\boldsymbol{Q}_{2}: L^{2}\left(\mathbb{R}^{3} ; \mathbb{R}^{3}\right) \rightarrow L^{2}\left(\mathbb{R}^{3} ; \mathbb{R}^{3}\right)$ has been shown in Sec. 3 to satisfy $\left\|\boldsymbol{Q}_{2}\right\|=1$ and using Lemma 4 , we have

$$
\left\|\boldsymbol{Q}_{2}^{\omega}\right\| \leq 1+2\left\|\boldsymbol{\nabla}\left(\mathcal{W}_{\omega}-\mathcal{W}\right)\right\| \leq 1+2 C_{2}(k R)^{2}
$$

Therefore, the convergence condition $\left\|\boldsymbol{Q}_{\omega}\right\|<1$ for fixed-point iterations is satisfied if

$$
\frac{|r|}{r+2}\left(1+2 C_{2}(k R)^{2}\right)<1 \quad \text { i.e. } \quad C_{2} r(k R)^{2}<h(r)
$$

(note that $r>-1$ and $r \neq 0$, implying $r+2>0$ and $0<h(r)<\infty$ in all cases).

Since the subset $\left\{(r, s) \mid r>-1, r \neq 0, s>0, r C_{1}>1, C_{2} s^{2}<h(r)\right\}$ of the real $(r, s)$-plane is non-empty, Prop. 1 implies that there are inhomogeneity configurations (characterized by $B, \rho^{\star}$ and $\omega$ ) for which fixed-point iterations converge for the modified SVIE (38) even though they diverge for the classical SVIE (37). Applying factorizations provided by (30), or by Lemma 1 in elasticity, to the frequency-domain singular integral operator therefore extends the range of applicability (in terms of frequency and scatterer characteristics) of fixed-point computational iterative methods.

5.2. Scattering of elastic waves. The analysis of Sec. 5.2 can be transposed in a straightforward way to the scattering of elastic waves by inhomogeneities such that $\Delta \mathcal{C} \neq \mathbf{0}$ and $\Delta \rho=0$ and embedded in isotropic media, yielding the elastodynamic counterpart of Prop. 1. This relies mainly on proving the counterparts of lemmas 3 and 4 . The elastodynamic version of the analytical solution used in Sec. 6.3 for the proof of Lemma 3 can be obtained using similar separation of variable methods and at the expense of heavier algebra. The counterpart of Lemma 4 (setting e.g. $k=\omega \sqrt{\rho / \mu}$ ) follows using the method of Sec. 6.3 , since the asymptotic behavior at the origin and at infinity of the elastodynamic fundamental tensor and its derivatives is similar to that of the acoustic fundamental solution.

\section{Auxiliary proofs.}

6.1. Proof of satisfaction of VIE by FSTP solutions. Consider a bounded domain $X \subset \mathbb{R}^{3}$, and let the partial differential operator $\mathcal{P}^{\prime}$ be defined (in the distributional sense) by $\mathcal{P}^{\prime} \boldsymbol{w}=-\operatorname{div}\left(\mathcal{C}^{\prime}: \boldsymbol{\varepsilon}[\boldsymbol{w}]\right)$ for some elasticity tensor $\mathcal{C}^{\prime}$. Let $\boldsymbol{w} \in H^{1}\left(X ; \mathbb{R}^{3}\right)$, and define the distribution $\widetilde{\boldsymbol{w}}$ as the extension of $\boldsymbol{w}$ by zero in $\mathbb{R}^{3} \backslash \bar{X}$. For any test function $\boldsymbol{\varphi} \in \mathcal{D}:=C_{0}^{\infty}\left(\mathbb{R}^{3} ; \mathbb{R}^{3}\right)$, one has

$$
(\mathcal{P} \widetilde{\boldsymbol{w}}, \boldsymbol{\varphi})=\left(\widetilde{\boldsymbol{w}},\left(\mathcal{P}-\mathcal{P}^{\prime}\right) \boldsymbol{\varphi}\right)+\left(\widetilde{\boldsymbol{w}}, \mathcal{P}^{\prime} \boldsymbol{\varphi}\right)=\left(\boldsymbol{w},\left(\mathcal{P}-\mathcal{P}^{\prime}\right) \boldsymbol{\varphi}\right)_{X}+\left(\boldsymbol{w}, \mathcal{P}^{\prime} \boldsymbol{\varphi}\right)_{X}
$$


(with $(\cdot, \cdot)$ and $(\cdot, \cdot)_{X}$ respectively denoting the distributional duality product and the $L^{2}(X)$ scalar product), since $\mathcal{P}$ involves only even-order derivatives. Next, using the definition of operators $\mathcal{P}^{\prime}$ and $\mathcal{P}$, applying the first and second Green identities [10, Thm. 4.4] for the domain $X$ to $\left(\boldsymbol{w},\left(\mathcal{P}-\mathcal{P}^{\prime}\right) \boldsymbol{\varphi}\right)_{X}$ and $\left(\boldsymbol{w}, \mathcal{P}^{\prime} \boldsymbol{\varphi}\right)_{X}$, respectively, and combining the resulting expressions, one finds

$$
(\mathcal{P} \widetilde{\boldsymbol{w}}, \boldsymbol{\varphi})=-(\boldsymbol{w}, \boldsymbol{t}[\boldsymbol{\varphi}])_{\partial X}+\left(\boldsymbol{t}^{\prime}[\boldsymbol{w}], \boldsymbol{\varphi}\right)_{\partial X}-\left(\left(\mathcal{C}^{\prime}-\mathcal{C}\right): \varepsilon[\boldsymbol{w}], \varepsilon[\boldsymbol{\varphi}]\right)_{X}+\left(\mathcal{P}^{\prime} \boldsymbol{w}, \boldsymbol{\varphi}\right)_{X}
$$

(where $\boldsymbol{\varphi} \rightarrow \boldsymbol{t}^{\prime}[\boldsymbol{\varphi}]$ denotes the surface traction operator relative to elastic properties $\mathcal{C}^{\prime}$, and having used the formal self-adjointness of $\mathcal{P}^{\prime}$ in the last term).

Equality (39) holds for arbitrarily chosen bounded domain $X \subset \mathbb{R}^{3}$ and admissible elasticity tensor $\mathcal{C}^{\prime}$. It is now applied (i) for $X=B$ with $\boldsymbol{w}=\boldsymbol{u}_{B}^{-}$and $\mathcal{P}=\mathcal{P}^{\star}$, (ii) for $X=B_{R} \backslash B$ with $\boldsymbol{w}=\boldsymbol{u}_{B}^{+}$and $\mathcal{P}^{\prime}=\mathcal{P}$, and (iii) for $X=B_{R}$ with $\boldsymbol{w}=\boldsymbol{u}$ and $\mathcal{P}^{\prime}=\mathcal{P}$ (where $B_{R}$ is the ball of radius $R$ centered at the origin, bounded by the sphere $S_{R}$ ), yielding the identities

$$
\begin{aligned}
\left(\mathcal{P} \widetilde{\boldsymbol{u}}_{B}^{-}, \boldsymbol{\varphi}\right) & =-\left(\boldsymbol{u}_{B}^{-}, \boldsymbol{t}[\boldsymbol{\varphi}]\right)_{\Gamma}+\left(\boldsymbol{t}^{\star}\left[\boldsymbol{u}_{B}^{-}\right], \boldsymbol{\varphi}\right)_{\Gamma}-\left(\Delta \mathcal{C}: \varepsilon\left[\boldsymbol{u}_{B}^{-}\right], \boldsymbol{\varepsilon}[\boldsymbol{\varphi}]\right)_{B}+\left(\mathcal{P}^{\star} \boldsymbol{u}_{B}^{-}, \boldsymbol{\varphi}\right)_{B}, \\
\left(\mathcal{P} \widetilde{\boldsymbol{u}}_{B}^{+}, \boldsymbol{\varphi}\right) & =\left(\boldsymbol{u}_{B}^{+}, \boldsymbol{t}[\boldsymbol{\varphi}]\right)_{\Gamma}-\left(\boldsymbol{t}\left[\boldsymbol{u}_{B}^{+}\right], \boldsymbol{\varphi}\right)_{\Gamma}-\left(\boldsymbol{u}_{B}^{+}, \boldsymbol{t}[\boldsymbol{\varphi}]\right)_{S_{R}}+\left(\boldsymbol{t}\left[\boldsymbol{u}_{B}^{+}\right], \boldsymbol{\varphi}\right)_{S_{R}}+\left(\mathcal{P} \boldsymbol{u}_{B}^{+}, \boldsymbol{\varphi}\right)_{B_{R} \backslash B} \\
-(\boldsymbol{P} \widetilde{\boldsymbol{u}}, \boldsymbol{\varphi}) & =(\boldsymbol{u}, \boldsymbol{t}[\boldsymbol{\varphi}])_{S_{R}}-(\boldsymbol{t}[\boldsymbol{u}], \boldsymbol{\varphi})_{S_{R}}-(\mathcal{P} \boldsymbol{u}, \boldsymbol{\varphi})_{B_{R}}
\end{aligned}
$$

(the sign inversion for integrals over $\Gamma$ in the second equality being caused by the unit normal to $\Gamma$ conventionally pointing inwards of $\left.\mathbb{R}^{3} \backslash \bar{B}\right)$. Summing those equalities, noting that $(2 \mathrm{a}, \mathrm{b})$ imply $(\mathcal{P} \boldsymbol{u}, \boldsymbol{\varphi})_{B_{R}}-\left(\mathcal{P}^{\star} \boldsymbol{u}_{B}^{-}, \boldsymbol{\varphi}\right)_{B}-\left(\mathcal{P} \boldsymbol{u}_{B}^{+}, \boldsymbol{\varphi}\right)_{B_{R} \backslash B}=0$ for any test function, and invoking the transmission conditions $(2 \mathrm{~d})$ gives

$$
\left(\mathcal{P}\left(\widetilde{\boldsymbol{u}}_{B}^{+}+\widetilde{\boldsymbol{u}}_{B}^{-}-\widetilde{\boldsymbol{u}}\right), \boldsymbol{\varphi}\right)=-\left(\Delta \mathcal{C}: \varepsilon\left[\boldsymbol{u}_{B}^{-}\right], \boldsymbol{\varepsilon}[\boldsymbol{\varphi}]\right)_{B}-\left(\boldsymbol{v}_{B}, \boldsymbol{t}[\boldsymbol{\varphi}]\right)_{S_{R}}+\left(\boldsymbol{t}\left[\boldsymbol{v}_{B}\right], \boldsymbol{\varphi}\right)_{S_{R}}
$$

(with $\boldsymbol{v}_{B}:=\boldsymbol{u}_{B}^{+}-\boldsymbol{u}$ ). Equality (40) holds for any test function $\boldsymbol{\varphi} \in \mathcal{D}$, so is an equality between two distributions, whose supports are compact. One can then take the distributional convolution of both members by the fundamental tensor $\boldsymbol{G}$, which satisfies $\mathcal{P} \boldsymbol{G}=\delta \boldsymbol{I}$, to obtain

$$
\left(\widetilde{\boldsymbol{u}}_{B}^{+}+\widetilde{\boldsymbol{u}}_{B}^{-}-\widetilde{\boldsymbol{u}}, \boldsymbol{\varphi}\right)=-\left(\Delta \mathcal{C}: \boldsymbol{\varepsilon}\left[\boldsymbol{G} \star \boldsymbol{u}_{B}\right], \boldsymbol{\varepsilon}[\boldsymbol{\varphi}]\right)_{B}-\left(\boldsymbol{v}_{B}, \boldsymbol{t}[\boldsymbol{G} \star \boldsymbol{\varphi}]\right)_{S_{R}}+\left(\boldsymbol{t}\left[\boldsymbol{v}_{B}\right], \boldsymbol{G} \star \boldsymbol{\varphi}\right)_{S_{R}}
$$

where the left-hand side results from $\boldsymbol{G} \star \mathcal{P} \boldsymbol{w}=\mathcal{P} \boldsymbol{G} \star \boldsymbol{w}=\boldsymbol{w}$, and the right-hand side from $(\boldsymbol{G} \star \boldsymbol{w}, \boldsymbol{\varphi})=(\boldsymbol{w}, \boldsymbol{G} \star \boldsymbol{\varphi})$, for any compactly supported distribution $\boldsymbol{w} \in \mathcal{D}^{\prime}\left(\mathbb{R}^{3} ; \mathbb{R}^{3}\right)$.

The remaining task is to evaluate each term in the right-hand side of (41). We have

$$
\begin{array}{r}
\left(\Delta \mathcal{C}: \varepsilon\left[\boldsymbol{G} \star \boldsymbol{u}_{B}\right], \boldsymbol{\varepsilon}[\boldsymbol{\varphi}]\right)_{B}=-\int_{\mathbb{R}^{3}}\left\{\int_{B} \boldsymbol{\nabla} \boldsymbol{G}(\boldsymbol{x}-\boldsymbol{y}): \Delta \mathcal{C}(\boldsymbol{y}): \nabla \boldsymbol{u}_{B}^{-}(\boldsymbol{y}) \mathrm{d} V(\boldsymbol{y})\right\} \cdot \boldsymbol{\varphi}(\boldsymbol{x}) \mathrm{d} V(\boldsymbol{x}) \\
=-\left(\mathcal{W}\left[\Delta \mathcal{C}: \varepsilon\left[\boldsymbol{u}_{B}^{-}\right]\right], \boldsymbol{\varphi}\right)
\end{array}
$$

Moreover, the assumed decay conditions (2c) for $\boldsymbol{v}_{B}$, together with the boundedness of $\operatorname{supp}(\boldsymbol{\varphi})$ and the known decay of $\boldsymbol{x} \mapsto \boldsymbol{G}(\boldsymbol{x}-\boldsymbol{y})$ for $\boldsymbol{y} \in \operatorname{supp}(\boldsymbol{\varphi})$, imply

$$
\lim _{R \rightarrow+\infty}\left(-\left(\boldsymbol{v}_{B}, \boldsymbol{t}[\boldsymbol{G} \star \boldsymbol{\varphi}]\right)_{S_{R}}+\left(\boldsymbol{t}\left[\boldsymbol{v}_{B}\right], \boldsymbol{G} \star \boldsymbol{\varphi}\right)_{S_{R}}\right)=0
$$

for any test function $\varphi \in \mathcal{D}$. Using (42) and (43) in (41), sending $R$ to infinity and noting that the resulting distributions are locally summable functions, we obtain

$$
\boldsymbol{u}_{B}-\boldsymbol{u}=\mathcal{W}\left[\Delta \mathcal{C}: \varepsilon\left[\boldsymbol{u}_{B}\right]\right] \quad \text { in }\left(\mathbb{R}^{3} \backslash \bar{B}\right) \cup B
$$


Concluding, any solution $\boldsymbol{u}_{B}$ of the FSTP $\left.2 \mathrm{a}-\mathrm{d}\right)$ satisfies the volume integrodifferential equation (10a) and the associated integral representation formula (10c).

6.2. Proof of Lemma 3. Let $B=B_{R}:=\left\{\boldsymbol{x} \in \mathbb{R}^{3},|\boldsymbol{x}|<R\right\}$. We establish the claimed lower bound of $\left\|\boldsymbol{\nabla} \mathcal{W}_{\omega}\right\|$ for $\boldsymbol{\nabla} \mathcal{W}_{\omega}: L^{2}\left(B_{R} ; \mathbb{R}^{3}\right) \rightarrow L^{2}\left(B_{R} ; \mathbb{R}^{3}\right)$ by explicitly computing $\boldsymbol{g}:=\boldsymbol{\nabla} \mathcal{W}_{\omega}\left[\boldsymbol{h}^{\star}\right]$ for some given $\boldsymbol{h}^{\star} \in L^{2}\left(B_{R} ; \mathbb{R}^{3}\right)$. A simple choice is $\boldsymbol{h}^{\star}=\boldsymbol{e}_{3}$ in $B$, for which $\operatorname{div} \boldsymbol{h}^{\star}=0$ in $B$. We then have $\boldsymbol{g}=\boldsymbol{\nabla} v$, where $v$ is the radiating solution to the transmission problem

$$
\left(\Delta+k^{2}\right) v=0 \quad \text { in } B \cup\left(\mathbb{R}^{3} \backslash \bar{B}\right), \quad v^{+}=v^{-} \text {and }\left.\partial_{n} v\right|_{+}=\left.\partial_{n} v\right|_{-}+\boldsymbol{e}_{3} \cdot \boldsymbol{n} \quad \text { on } \partial B,
$$

which is the counterpart of problem (26) for the Helmholtz equation. This problem can easily be solved analytically, using spherical coordinates, yielding

$$
v(\boldsymbol{x})=k^{-1} E(k R) j_{1}(k|\boldsymbol{x}|)(\boldsymbol{x} /|\boldsymbol{x}|) \cdot \boldsymbol{e}_{3} \quad\left(\boldsymbol{x} \in B_{R}\right), \quad \text { with } \quad E(z):=-\mathrm{i} z^{2} h_{1}(z)
$$

$\left(j_{m}\right.$ and $h_{m}$ denoting, respectively, the spherical Bessel and Hankel functions of first kind and order $m$, see e.g. [14, Chap. 10]). The $L^{2}$ norm of $\boldsymbol{\nabla} v$ can then also be explicitly derived (with the help of identity $\left(z j_{1}^{2}(z)\right)^{\prime}=2 z j_{0}(z) j_{1}(z)-3 j_{1}^{2}(z)$ and classical interrelations between the $\left.j_{m}\right)$, and we find

$$
\|\boldsymbol{\nabla} v\|_{L^{2}\left(B_{R}\right)}^{2}=\frac{4 \pi}{3 k^{3}}|E(k R)|^{2} f(k R), \quad \text { with } \quad f(z):=\frac{1}{2}\left(z-\cos z \sin z-4 z j_{1}^{2}(z)\right) .
$$

Since $\left\|\boldsymbol{h}^{\star}\right\|_{L^{2}\left(B_{R}\right)}^{2}=4 \pi R^{3} / 3$, we then have

$$
\left\|\boldsymbol{\nabla} \mathcal{W}_{\omega}\right\|^{2} \geq \frac{\|\boldsymbol{\nabla} v\|_{L^{2}\left(B_{R}\right)}^{2}}{\left\|\boldsymbol{h}^{\star}\right\|_{L^{2}\left(B_{R}\right)}^{2}}=F(k R), \quad \text { with } \quad F(z):=z^{-3}|E(z)|^{2} f(z) .
$$

The well-known small-argument and large-argument asymptotic limiting forms of $j_{1}$ and $h_{1}$ (see e.g. [14, Chap. 10]) allow to show that

$$
\lim _{z \rightarrow 0} F(z)=1 / 9, \quad \lim _{z \rightarrow \infty} F(z)=1 / 2 .
$$

Since $F(z)>0$ for any $z>0$, the above limits imply that $z \mapsto F(z)$ has a strictly positive minimum $F_{\min }\left(\right.$ as $\left|h_{1}(z)\right| \neq 0$ implies $|E(z)|^{2}>0$, while $f^{\prime}(z)=2 j_{1}^{2}(z)+\left(z j_{0}(z)-2 j_{1}(z)\right)^{2}>0$ and $f(0)=0$ imply $f(z)>0$ for $z \neq 0$ ), and Lemma 3 holds true with $C_{1}:=\sqrt{F_{\min }}$; moreover $C_{1}$ is independent of $\omega$ and $R$ since $F$ depends only on the non-dimensional variable $z$. Numerical evidence indicates that $F_{\min }=F(0)=1 / 9$.

6.3. Proof of Lemma 4. The tensor-valued function $\boldsymbol{r} \mapsto \boldsymbol{\nabla}\left(G_{\omega}-G\right)(\boldsymbol{r})$ is known to have a $O\left(|\boldsymbol{r}|^{-1}\right)$ singularity at the origin and to be $C^{\infty}\left(\mathbb{R}^{3} \backslash\{\mathbf{0}\}\right)$; therefore $(\boldsymbol{x}, \boldsymbol{y}) \mapsto \boldsymbol{\nabla} \nabla\left(G_{\omega}-G\right)(\boldsymbol{y}-$ $\boldsymbol{x}) \in L^{2}\left(B_{R} \times B_{R} ; \mathbb{R}^{3 \times 3}\right)$. An upper bound of the norm of $\boldsymbol{\nabla}\left(\mathcal{W}_{\omega}-\mathcal{W}\right): L^{2}\left(B_{R} ; \mathbb{R}^{3}\right) \rightarrow L^{2}\left(B_{R} ; \mathbb{R}^{3}\right)$ is thus provided by the Hilbert-Schmidt norm

$$
\left\|\nabla\left(\mathcal{W}_{\omega}-\mathcal{W}\right)\right\|_{\mathrm{HS}}^{2}:=\int_{|\boldsymbol{x}|<R} \int_{|\boldsymbol{y}|<R}\left|\nabla \nabla\left(G_{\omega}-G\right)(\boldsymbol{y}-\boldsymbol{x})\right|^{2} \mathrm{~d} V(\boldsymbol{x}) \mathrm{d} V(\boldsymbol{y}) .
$$

Now, using definition (36) of $G_{\omega}$ and the homogeneity properties (5) of $G$, it is straightforward to check that $\boldsymbol{\nabla} \nabla\left(G_{\omega}-G\right)(\boldsymbol{r})=k^{3} \boldsymbol{\nabla} \nabla(g-G)(k \boldsymbol{r})$, with the function $g$ defined by $g(\boldsymbol{z})=e^{\mathrm{i}|\boldsymbol{z}|} /(4 \pi|\boldsymbol{z}|)$. Setting $\boldsymbol{x}=k^{-1} \overline{\boldsymbol{x}}$ and $\boldsymbol{y}=k^{-1} \overline{\boldsymbol{y}}$ in the above double integral, we obtain

$$
\left\|\boldsymbol{\nabla}\left(\mathcal{W}_{\omega}-\mathcal{W}\right)\right\|_{\mathrm{HS}}^{2}=\int_{|\overline{\boldsymbol{x}}|<k R} \int_{|\overline{\boldsymbol{y}}|<k R}|\nabla \nabla(g-G)(\overline{\boldsymbol{y}}-\overline{\boldsymbol{x}})|^{2} \mathrm{~d} V(\overline{\boldsymbol{x}}) \mathrm{d} V(\overline{\boldsymbol{y}}) .
$$


Moreover, since $\boldsymbol{r} \mapsto|\boldsymbol{r}| \boldsymbol{\nabla} \boldsymbol{\nabla}(g-G)(\boldsymbol{r})$ is bounded at the origin and at infinity while being $C^{\infty}\left(\mathbb{R}^{3} \backslash\{\mathbf{0}\}\right)$, there exists a constant $E>0$ such that

$$
|\boldsymbol{\nabla} \nabla(g-G)(\boldsymbol{r})| \leq E|\boldsymbol{r}|^{-1} \quad \text { for all } \boldsymbol{r} \in \mathbb{R}^{3} \backslash\{\mathbf{0}\},
$$

with the best (i.e. smallest) value of $4 \pi E$ found numerically to be approximately given by $4 \pi E \approx 1.23154$. We therefore have

$$
\begin{array}{r}
\left\|\boldsymbol{\nabla}\left(\mathcal{W}_{\omega}-\mathcal{W}\right)\right\|_{\mathrm{HS}}^{2} \leq E^{2} \int_{|\overline{\boldsymbol{x}}|<k R} \int_{|\overline{\boldsymbol{y}}|<k R}|\overline{\boldsymbol{y}}-\overline{\boldsymbol{x}}|^{-2} \mathrm{~d} V(\overline{\boldsymbol{x}}) \mathrm{d} V(\overline{\boldsymbol{y}})=(k R)^{4} E^{2} D^{2}, \\
\text { with } D^{2}:=\int_{|\boldsymbol{\xi}|<1} \int_{|\boldsymbol{\eta}|<1}|\boldsymbol{\xi}-\boldsymbol{\eta}|^{-2} \mathrm{~d} V(\boldsymbol{\xi}) \mathrm{d} V(\boldsymbol{\eta})=4 \pi^{2}
\end{array}
$$

(where the value $2 \pi$ of $D$ is found by analytical evaluation of the double integral), implying that Lemma 4 holds true with $C_{2}:=2 \pi E \approx 0.61577$.

\section{REFERENCES}

1. Bonnet M. Solvability of a volume integral equation formulation for anisotropic elastodynamic scattering. J. Integral Eq. Appl., 28:169-203 (2016).

2. Dassios G., Karadima K.S. Time harmonic acoustic scattering in anisotropic media. Math. Meth. in Appl. Sc., 28:1383-1401 (2005).

3. Eshelby J.D. The determination of the elastic field of an ellipsoidal inclusion and related problems. Proc. Roy. Soc. A, 241:376-396 (1957).

4. Freidin A.B., Kucher V.A. Solvability of the equivalent inclusion problem for an ellipsoidal inhomogeneity. Math. Mech. Solids, 21:255-262 (2016).

5. Gintides D., Kiriaki K. Solvability of the integrodifferential equation of Eshelby's equivalent inclusion method. Quart. J. Mech. Appl. Math., 68:85-96 (2015).

6. Helnwein P. Some remarks on the compressed matrix representation of symmetric second-order and fourthorder tensors. Comp. Meth. Appl. Mech. Eng., 190:2753 - 2770 (2001).

7. Hsiao G.C., Wendland W.L. Boundary integral equations. Springer (2008).

8. Kupradze V.D. (editor). Three-dimensional problems of the mathematical theory of elasticity and thermoelasticity. North Holland (1979).

9. Kuykendall W.P., Cash W.D., Barnett D.M., Cai W. On the existence of Eshelby's equivalent ellipsoidal inclusion solution. Math. Mech. Solids, 17:840-847 (2012).

10. McLean W. Strongly elliptic systems and boundary integral equations. Cambridge (2000).

11. Mikhlin S.G., Prössdorf S. Singular integral operators. Springer-Verlag (1986).

12. Mura T. Micromechanics of Defects in Solids. Martinus Nijhoff (1987).

13. Natroshvili D. Boundary integral equation method in the steady state oscillation problems for anisotropic bodies. Math. Meth. in Appl. Sc., 20:95-119 (1997).

14. Olver F.W.J., Lozier D.W., Boisvert R.F., Clark C.W. (editors). NIST handbook of mathematical functions. Cambridge (2010).

15. Pankratov O.V., Kuvshinov A.V., Avdeev D.B. High-performance three-dimensional electromagnetic modelling using modified Neumann series. Anisotropic Earth. J. Geomag. Geoelectr., 49:1541-1547 (1997).

16. Potthast R. Electromagnetic scattering from an orthotropic medium. J. Integral Eq. Appl., 11:197-215 (1999).

POems (ensta Paristech, CNRS, inRIA, Université Paris-Saclay), 91120 Palaiseau, France. MBONNET@ENSTA.FR 